\title{
Communication, Collaboration \& Trust: Interpersonal Challenges in Virtual Collaboration Team
}

\author{
Dr. Pooja Raj Srivastava
}

Department of Humanities, Vidyalankar Group of Institutes, Mumbai, Maharashtra, India

\begin{abstract}
The workplace and the working pattern both are going through continual transformation. To prevail over the challenges of the current scenario the organizations, whether companies or academic institutes, have shifted their working patterns and have embraced 'Remote Work'.

Collaborative technologies are at the center of this Trans mutated state. These collaborative technologies help organizations to continue collaborations, in the form of computer-aided, synchronous or asynchronous collaboration and help to create dynamic work environments. Team members share responsibilities as a team for outcomes but accomplish their task in an independent way. They rely on mediated form of communication rather than face-to-face as they are geographically dispersed. The team produce an outcome in the form of shared understanding, strategy planning, evaluation methods, recommendation, decision making, and action plan related to a task or other responsibilities.

Despite considerable developments in Information Communication Technology (ICT) over the period, Virtual Collaborative Teams (VCT) still confront multiple interpersonal and communication challenges. VCTs need additional attention from both corporations and academics in order to successfully implement and reap the benefits of their virtual operations.
\end{abstract}

Keywords—virtual collaboration, team, work, shared responsibilities, challenges, interpersonal

\section{INTRODUCTION}

Changing scenario and viability of organizational work enforced to think about the alternative sources that help to continue the organizational work. Rapid technological advancements and changing global demands over the past decades has changed the nature of work within organizations (Bell \& Kozlowski, 2002). Virtual teams (VT) and collaborations are not a new phenomenon now a days, many organizations, especially MNCs (restricted to certain organizations, where global work pattern prevail) have such sort of work methodology since long time, yet the changing global demands and situational crisis (when social distancing and staying at home is the best way to avoid pandemic situation) enforced the alternate. Currently, virtual collaboration is the modus operandi of almost all the organizations. The technological advancements help to execute the organizational work and expedite such changes.
Scholars claim an increased number of corporations have shifted its operations into a virtual setting to face new demands. (Alsharo, Gregg \& Ramirez, 2017).

Advancements related to communication technology offer flexibility to the employees to conduct work from home and even communicate across remote geographical distances (Bergiel, \& Balsmeier, 2008). VTs consist of individuals working independently towards a shared goal, whilst operating geographically dispersed, regardless of time and space (Batarseh, Daspit \& Usher, 2017). VCTs are becoming increasingly the norm in organizational structures. This enables team members to work simultaneously on the same project assignments without the need of proximity, which leads to increased...efficiency (Zuofa \& Ochieng, 2017.) As such, virtual teams allow organizations to bring together people with the best expertise, regardless of where they live (Bell \& Kozlowski, 2002). VCTs allow faster and shared 
decision-making processes and adaption to dynamic market changes, whilst simultaneously improving business operations (Valacich \& Schneider, 2017).

The three associated aspects of the term VT are- virtual, team and technology that elaborate the concept explicitly. Virtual implicates various features such as physical locations, time zones, and the other compositional aspects. Team signifies structure, size, operational methodologies, procedures and reporting relationship (hierarchical pattern). And Technology signifies the tools, mode and platform of communication. Organizations employ various action plans to attain organizational goals, cater individual's needs, foster relationship building, communication, strengthen collaboration, and improve technological skills, in order to succeed in VCTs. The team... work together with the help of various applications, e.g., chat rooms, e-mail, instant messaging, video conferencing, etc. using collaborative technology in order to achieve organizational goals.

The resultant developments in the field of ICT catered the attention of organizations regarding employability of remote work. Thus, various scholars therefore maintain that ICT is the core pillar of a successful VCT (Wildman \& Griffith, 2015). Information and communications technologies (ICTs): Technology devices enabled organizations and individuals to handle business operations anywhere and anytime, that include video conferencing, telephones, teleconferencing, Skype, instant messaging, webcam, and emails. The role of telecommunication technologies, especially ICT is unquestionable in Virtual collaborations. ICT is argued to enable easier, faster and more cost-efficient ways of establishing connectivity across business operations (Martinic, Fertalj \& Kalpic, 2012). The various online tools that facilitate in virtual collaborations such as audio \& video conferencing, web-based or computer-aided media etc. are the dominant forms of communication.

VCTs are now an integral and vital part of organizational operations; however, various challenges impede the functioning and it becomes difficult for organizations to overcome in order to exercise VCTs in a successful manner. Although ICT facilitated implementation of VCTs, questions remain regarding functionality and efficiency in the virtual context in which corporations face multiple communication challenges (Marlow et al., 2018).

This study focuses on the issues related to optimum employability of virtual collaboration by constructing the approach at different levels. It highlights the challenges and also suggests the measures to improve the productivity of virtual teams. Chang, Chuang and Chao (2011) mentioned that highlighting the importance of investigating virtual communication in multiple settings and further gaining a deeper understanding of the factors which obstruct efficient VCT communication...can help to develop the virtual working pattern in any organization. Explicitly, the study illustrates and focuses on the three major issues communication, collaboration and trust that relates itself to the interpersonal factors in virtual teams. The understanding of the said challenges and suggestive measures can help to accentuate the collaborations in VTs.

\section{CHALLENGES OF VIRTUAL COLLABORATION TEAM}

Despite significant progress in ICT over the period, VTs still face multiple challenges. Most of the organizations are still in the inception of implementing ICT; therefore, developing insights into the challenges of VTs is crucial. Deloitte (2018) exhibits the convolution of VTs by a report in which 1600 executives elaborates how ICT offered enormous opportunities to organizations, while also discussed the great deal of uncertainty and challenges associated. One main cause of VCT complexity is the paucity of a best practice, with no one-size-fits-all type of concept applicable to complex situations (McKinsey, 2015). Geographically dispersed members of the team face difficulty to develop team coherence and team efficiency. Daim et al. (2012) analyzed a framework to illustrate the following challenges for communication in a virtual context namely: Technology, Interpersonal Relations, Trust, Leadership and Cultural Differences. Most of these challenges are related to the process involved in interpersonal relations like communication, trust and collaboration.

Efficient communication is argued to be one core of establish, maintaining and creating trust and team collaboration in virtual settings (Kelly, 2013). Due to lack of face-to-face communication, the virtual teams completely rely on virtual communication hence a well-equipped technological infrastructure is therefore of crucial importance for VCT (Kankanhalli, Tan \& Wei, 2014). Daim et al. (2012) describes the importance of technology in VCT: "Without Internet, email, video conference and audio bridges, virtual teams can't even exist" (p. 200). Corporations need to get updated with the continual change and emerging forms of communication; the new trends such as mobile \& social media platforms, cloud- 
based computing, artificial intelligence and big data structures (PwC, 2018.). Hence, updated and modernize technology becomes a critical and continuous complexity for any corporation to possess. Therefore, Daim et al. (2012) argue a common negative experience for members in VCT is technological failure. Batarseh, Daspit and Usher (2017) specify that only $18 \%$ of all VTs achieve communicative success. The ensuing outcome of the signal failure can be in the form of delayed or failing audio or video signals, resultantly the group members find difficulty in virtual collaborations, e.g. unable to understand the context, disruptions in communication and thereby creating disturbances between the members of the team. Failures in virtual communication can result in misunderstandings, limit team communication and overall decreased team productivity (Sallnäs, 2005).

The importance of face-to-face communication for VTs is undeniably excessive (Alsharo, Gregg \& Ramirez, 2017). In virtual communication, the dispersed members cannot discern physical behaviors and cues, which can disrupt the establishment of inter-personal relations. The lack of face-toface communication imply difficulties in achieving correspondence and the assistance of maintaining relationships (Daim et al., (2012). According to the reportThe challenges of working in Virtual Teams: Virtual Teams Survey Report (2010), Virtual teams differed most from faceto-face teams in three areas: managing conflict $(73 \%)$, making decisions (69\%), and expressing opinions (64\%)" (p 3). Respondents cited that these three are interrelated and challenging for virtual teams. In the absence of an ability to express opinions or manage conflicts among members, virtual teams find it challenging to make decisions. The most common sources of conflict were miscommunication of information $(33 \%)$ and inconsistent expectations $(21 \%)$ of quality or team norms. Harzing et al. (2011) states that differences of language complicates and slow down decisionmaking processes and affect the interpersonal relation of VTs.

In the virtual context, the non-verbal communication such as gestures, facial expression and body language etc. is certainly difficult to peruse, compared to face-to face communication. The misinterpretations resultantly obstruct or disrupt the comprehension of the information and develop misunderstandings among team members (Wang, 2009). Daim et al. (2012) further explains limited successful communication and misinterpretations could lead to confusion and decreased team performance due to miscommunication among team members. The said report -The challenges of working in Virtual Teams: Virtual Teams Survey Report (2010) also stated that respondents indicated that the greatest virtual challenge they faced was inability to read non-verbal cues. "Not surprisingly, our studies and anecdotal evidence indicated that the inability to read body language (which according to some studies represents $70 \%$ of the message) led to the biggest challenges that virtual teams face. In this survey, the inability to read non-verbal cues was a challenge of varying degree to $94 \%$ of all team members. Furthermore, it is closely linked to the lack of face-to-face meetings: we found that $46 \%$ of virtual team members never meet in person." ( $p$ 12).

Trust is another factor that argued to be vital in VTs for successful compilation of business operations. Teams operating in a virtual setting are required to trust its team members to perform efficiently. Interactions, inter-personal relations and collaborations help to build up Trust in the team. Trust is interconnected with creating personal relationships, which is to be improved through continuous interactions between individuals over time (Soetanto, 2012). But in VTs, as already mentioned, the absence of these essential elements makes it difficult. Scholars argue, teams, with lack of trust, tend to have more conflicts and inefficiency compared to teams with a high degree of trust (Soetanto, 2012). Trust is therefore an essential factor as it chains the connectivity among team members virtually and is therefore harder to establish (Zuofa \& Ochieng, 2017). The concerns related to trust issue in VTs seriously affect the successful implantation of the procedures and policies. Alsharo, Gregg and Ramirez (2017) claim trust to be crucial for creating and maintaining relationships, supporting teamwork and collaboration. Moreover, trust is described to be a fundamental element in projects and an important factor for team collaboration and successful team performance (Zuofa \& Ochieng, 2017; Soetanto, 2012). Batarseh, Daspit and Usher (2017). Given this importance, establishing trust is argued to be one great challenge for virtual collaboration (Marlow, Lacerenza \& Salas, 2017).

Collaboration is an important goal to ensure a successful working arrangement and accomplishments of the tasks for any organization. But virtual collaboration comes with its own unique challenges and difficulties. The problems that occur due to the virtual platform relates itself to the complexity of creating a relationship and establishing collaboration within a VCT (Daim et al., 2012). Researches have proven that people interaction is comparatively less in virtual communication, therefore lacks human interaction and inter-personal relations. 
Virtual communication is argued to have a negative impact on social interaction as the members interact less in virtual contexts. According to a survey by RW Culture Wizard (2016) $92 \%$ argue face-to-face communication to be the most effective form of communication. In comparison, internal and external social media tools for communication, such as instant messages or email, only score $49 \%$ on average providing an effective output. Various studies have denoted the fact that it is comparatively difficult to develop bond in virtual teams, and interact with genuine dialogue, and escalation of misunderstanding is easier.

Interpersonal relations play a crucial role in the development of collaboration of activities and accomplishments of designated tasks in VTs. It includes differences in group compositions, individual background and traits which are the differences impacting the creation of team collaboration. Hertel, Geister \& Konradt (2005). Thus, the significant element is to highlight the importance of being aware of our subjective differences as humans, as it adversely affect team collaboration and productivity if not managed and understood properly in VTs. Moreover, the teams are unique and need an altogether different approach to develop and establish rapport that lead to a positive outcome. The virtual teams can have a diverse composition, therefore developing collaboration and rapport may be arduous in absence of essential planning. The composition of VTs is generally diverse. The people with such a diverse background have subjective opinions and notions about working methodologies. This subjectivity refers to the opinion, in the form of assumed notions, disposed mindset, or procured inferences. Therefore, VTs must ensure the idea of team members and required to constantly effort to maintain and establish interpersonal relations, as it can be lost quickly if managed poorly (RW Culture Wizard, 2016).

\section{HOW TO OVERCOME THE CHALLENGES}

The uniqueness of virtual teams includes specific challenges such as project coordination, building relationships, and teamwork that are different from collocated teams (Mancini, 2010). Organizations certainly need to exercise the additional attentive measures to successfully implement and attain the benefits of virtual operations. To overcome the challenges, it is required to take a note of the challenges faced by VTs and then try to reconcile those challenges.

Team effectiveness is a multidimensional concept that includes several outcomes such as task performance, additional task behaviors, completing tasks promptly, and satisfaction (Webber, 2008). A relationship exists between virtual teams and the overall performance of organizations, which includes increase productivity and efficiency (Eissa et al., 2012; Palanski et al., 2011). The team leader plays a very important role in team building and hence hiring the right person is must. It is important to invest time during team setup. Adequate time should be invested for the clarification of team norms, goals and work codes. Explicit information in the form of written guidelines that illustrates the content, medium and expected behavior of the team members help to communicate to avoid ambiguity and misconception.

Communication need to be clear, effective, complete, fluent and on time, which can be complex to achieve if one of the requirements are not fulfilled (Marlow et al., 2018). Therefore, it is important to develop communication environment and foster a culture of communication throughout the organization. The Leader can set the example by providing regular updates and ensuring continuity of holding check-ins with the team. The team members reciprocate and follow the team leader by providing continuous feedback and maintaining touch. "Willingness to share information (18\%), followed by being proactively engaged (17\%), collaborative (17\%), organized (14\%), having good social skills (13\%), providing useful feedback (11\%), and offering assistance to teammates (10\%) are the characteristics that made good virtual teammates. The participants stated other important characteristics of a good virtual team in the form of -communication and language; coordination, technology, and a sense of collaboration; management, goals, and accountability; cultural bias, and better team selection." (The Challenges of working in Virtual Teams: Virtual Teams Survey Report, 2010).

Effective collaboration helps teams to develop bond and build trust and get acquainted with one another's thought processes and working styles. By using functional and potent virtual communication modes, VCTs operations could enhance team collaboration and team efficiency (Marlow, Lacerenza \& Salas, 2017). First, it helps to form clearly defined teams in the form of role assertion and outcome expectation. VTs can foster collaboration by establishing a mission statement. These broader organizational goals help to develop the feeling of cohesion by associating themselves to the defined mission statement. This helps to ascertain that people will work collaboratively with the clarity of roles assigned, even from a distance.

The other approach for optimized collaboration is to ensure accountability without invading privacy, the best way to 
ensure that everyone is doing their job is to set clear expectations for each role and have regular check-ins to gauge progress. Communicating expectations to team help them to envision the role assigned and shared goal. There should be a general idea of how long tasks take and how much effort is required to accomplish the task at individual level. The deadline must be in accordance with the level of complexity, size and execution of the work. The clarity and transparency are required at the part of the process of monitoring the progress. The said process is essential to foster productivity, transparency, and collaboration.

Other important point of consideration is choosing the best tool and platform that optimizes the communication. Communication has become an issue in virtual teams dealing with different communication technologies, tools, and the procedures for using the tools (Brandt et al., 2011). The team must understand the purpose of the communication and selecting the right medium accordingly. As every tool is not going to suit your purpose and be a good fit for your mode of communication, clarifying the purpose help to determine the best tool which strengthens the collaboration. Virtual teams predominantly use computer-mediated technology for communication. Therefore, there is a need for virtual team members to be knowledgeable in computer-mediated communication technology (Carlson et al., 2013). There are various tools that can be used according to the purpose of the meeting. Various applications like Slack, Twist, and Google Hangouts are good for chat. For Project management: Trello, Jira, Asana are there. Google Meet, Zoom, Cisco and WebEx assist in web and video conferencing, For Collaboration and prototyping, organizations prefer Invision, Marvel, Adobe XD. Calendly, Doodle is used for scheduling. And Workflow automation is best optimized with the help of Zapier, Microsoft Flow, Monday, etc. After selecting the best resource tool, it is important that users are acquainted with the functioning of those software programs. Hence, it is important to provide training to the members on the selected tool to ensure the better productivity and the fullest benefit.

\section{CONCLUSION}

Virtual communication is argued to be crucial for firm competitiveness on a global scale, allowing interaction across businesses' operations. The aim of this article is to investigate what communication challenges VTs face in a virtual setting and the various measures that can help to overcome the same. The study findings highlight several strategies that can be employed for successful employability of virtual team that leaders can use to build and foster communication, collaboration and trust among virtual team members to improve performance. Strategic organization and planning can help to set clear and attainable goals. Building interpersonal relations help to accelerate work culture in VTs. Hence, strategic approach is required for potential outcome.

\section{REFERENCES}

[1] Alsharo, Gregg, D. and Ramirez, R. (2017). "Virtual Team Effectiveness: The Role of Knowledge Sharing and Trust" Information \& Management, 54 (4), pp.479-490.

[2] Batarseh, F., Daspit, J. and Usher, J. (2016). "The Collaboration Capability of Global Virtual Teams: Relationships with Functional Diversity, Absorptive Capacity, and Innovation" International Journal of Management Science and Engineering Management, 13(1),pp.1-10.

https://www.bcg.com/publications/2016/strategyglobalization-saving-globalizationtechnology-fromthemselves.aspx

[3] Blomqvist, P., Oscar N. (2018). "Global Virtual Team Communication - An exploratory study on what challenges teams face in a virtual setting", Pp.1-72 https://www.divaportal.org/smash/get/diva2:1241189/FULLTEXT01.pdf

[4] Brandt, V., England, W., \& Ward, S. (2011) "Virtual Teams. Research Technology Management”, 54(6), 62-63. http://www.iriweb.org

[5] Carlson, J. R., Carlson, D. S., Hunter, E. M., Vaughn, R. L., \& George, J. F. (2013). "Virtual Team Effectiveness: Investigating the moderating role of experience with computer-mediated communication on the impact of team cohesion and openness", Journal of Organizational and End User Computing, 25(2), 118.

[6] Chang, H., Chuang, S. and Chao, S. (2011). "Determinants of Cultural Adaptation, Communication Quality, and Trust in Virtual Teams' Performance", Total Quality Management \& Business Excellence, 22(3), pp.305-329.

[7] Daim, T., Ha, A., Reutiman, S., Hughes, B., Pathak, U., Bynum, W. and Bhatla, A. (2102). "Exploring the Communication Breakdown in Global Virtual Teams", International Journal of Project Management, 30(2), pp.199-212.

[8] Deloitte. (2018). "The Fourth Industry Revolution is here - Are you ready?" https://www.forbes.com/forbes-insights/ourwork/fourth-industrial-revolution/

[9] Eissa, G., Fox, C., Webster, B. D., \& Kim, J. (2102). "A Famework for Leader Effectiveness in Virtual Teams", Journal of Leadership, Accountability \& Ethics, 9 (2), 11-22. http://www.na-businesspress.com/jlaeopen.html

[10] Harzing, A., Köster, K. and Magner, U. (2011). "Babel in Business: The language barrier and its solutions in the HQ- 
subsidiary relationship", Journal of World Business, 46(3), pp.279-287.

[11] Hertel, G., Geister, S. and Konradt, U. (2005). "Managing Virtual Teams: A review of current empirical research", Human Resource Management Review, 15(1), pp.69-95.

[12] Kankanhalli, A., Tan, B. and Wei, K. (2014). "Conflict and Performance in Global Virtual Teams", Journal of Management Information Systems, 23(3), pp.237-274.

[13] Kelly, N. (2013). "Building Trust in Communications" http://www.trustacrossamerica.com/documents/building-trust reports/KellyCommunications.pdf

[14] Mancini, D. J. (2010). "Building Organizational Trust in Virtual Teams", Journal of Behavioral Studies in Business, 2, 1-5, 2. http://www.aabri.com/jbsb.html

[15] Marlow, S., Lacerenza, C., Paoletti, J., Burke, C. and Salas, E. (2018). "Does team communication represent a one-size-fits-all approach? A meta-analysis of team communication and performance", Organizational Behavior and Human Decision Processes, 144, pp.145-170.

[16] Marlow, S., Lacerenza, C. and Salas, E. (2017). "Communication in Virtual Teams: A Conceptual Framework and Research Agenda", Human Resource Management Review, 27(4), pp.575-589.

[17] Martinic, A, K. Fertalj, and D. Kalpic. (2012). "Methodological Framework for Virtual Team Project Management", International Journal of Innovation, Management and Technology, 3(6), pp. 702-707.

[18] McKinsey. (2015). Can we talk? Five tips for communicating in turnarounds. $\quad$ https://www.mckinsey.com/businessfunctions/strategy-and-corporate-finance/ourinsights/can-wetalk-five-tips-for-communicating-in-turnarounds

[19] Powell, J. (2016). RW "Culture Wizard: Trends in Global Virtual Teams". http://cdn.culturewizard.com/PDF/Trends_in_VT_Report_417-2016.pdf

[20] R. Shelly. (2012). Virtual Collaboration: The Skills needed to Collaborate in a Virtual Environment, Journal of Internet Social Networking \& Virtual Communities, pp.1-8. https://ibimapublishing.com/articles/JISNVC/2012/629512/62 9512.pdf

[21] Sallnas, E. (2005). "Effects of Communication Mode on Social Presence, Virtual Presence, and Performance in Collaborative Virtual Environments. Presence: Teleoperators and Virtual Environments", 1(6), pp.434-449.

[22] Soetanto, R. (2012). "Communication modes and performance of virtual design teams in an undergraduate building project", Proceedings of the 28th Annual Conference of Association of Researchers in Construction Management, Edinburgh, pp. 177 18759.

[23] Valacich, J. and Schneider, C. (2017). "Information Systems Today”, Harlow, United Kingdom: Pearson Education Limited.

[24] Virtual Teams Survey Report. (2010). "The Challenges of Working in Virtual Teams”. R.W. Culture Wizard, pp.1-31. http://www.communicationcache.com/uploads/1/0/8/8/108872 48/the_challenges_of_working_in_virtual_teams.pdf

[25] Wainfan, Lynne. Davis, P.K. (2004). "Challenges in Virtual Collaboration: Videoconferencing, Audioconferencing and Computer-Mediated Communications". Rand Corporations.

[26] Wang, H. (2009). "Nonverbal Communication and the Effect on Interpersonal Communication", Asian Social Science, 5(11).

[27] Wildman, J. and Griffith, R. (2015). "Leading Global Teams", New York, NY: Springer.

[28] Zuofa, T. and Ochieng, E. (2017). "Working separately but together: appraising virtual project team challenges", Team Performance Management: An International Journal, 23(5/6), pp.227-242. 